\title{
Optimization Design of Network Structure for WSN Data Collection Design Based on Delay Perception
}

\author{
Wen Xiongjun ${ }^{1}$, Liu Yingchun ${ }^{1}$ and Liu Shukun ${ }^{1}$ \\ ${ }^{1}$ School of Information Science and Engineering, Hunan International Economics \\ University, Changsha, China, 410205
}

\begin{abstract}
In order to solve such problems as large delay and large energy consumption in current wireless sensor network (WSN) during data collection, a network structure for WSN data collection based on delay perception is designed in this article. Firstly, the tree structure thought is introduced therein to divide WSN sensor nodes into multiple single-layer clusters with different sizes so as to construct a new network structure, thus to improve the topological structure and enable the cluster heads to complete the data communication in an interlaced mode as well as significantly reduce the delay during data collection; secondly, the model for calculating the delay and the energy consumption during WSN data collection is established in this article; thirdly, Top-Down technology is introduced therein to design the network structure formation algorithm, thus to optimize the energy consumption of the data collection mechanism through the communication distance minimization. The experiment shows: compared with existing network structure for WSN data collection, the proposed network structure can not only effectively reduce the delay during WSN data collection, but also maintain the whole communication energy consumption at a relatively low level.
\end{abstract}

Keywords: Wireless Sensor Network; Data Collection; Network Topology; Communication Distance; Delay Perception; Energy Consumption

\section{Introduction}

In current WSN application, the sensors thereof are powered by batteries, thus causing the energy consumption of WSN nodes to become a difficult problem [1-2]. Hereto, Z Yang [3], et al. have designed WSN data collection protocol based on spatial clustering to reduce the energy consumption of WSN nodes and realize timeliness, and the corresponding experiment result shows that this algorithm can significantly prolong the network life cycle and greatly reduce the energy consumption of the nodes. H Bagci, A Yazici, et al. [4] have designed fuzzy energy aware unequal clustering algorithm to generate the clusters with different sizes, and the test data show that the algorithm has good stability and low energy consumption, but it is difficult to control the cluster quantity and the protocol will introduce additional energy consumption and delay the data collection during the data outputting process when the in-network data fusion fails to reduce any data. M Gupta, et al. [5] have proposed the link based data collection protocol, and the simulation result shows that the protocol can significantly reduce WSN communication energy consumption, but the head node of the protocol is a severe bottleneck and has large delay[6]. Ren Xiuli, et al. [7] have proposed a tree-form WSN data collection method for mobile base stations, and the experiment data shows that the algorithm can significantly improve network communication performance, but the method has large delay during data collection.

In order to solve such problem as large delay during WSN data collection, the network structure for WSN data collection based on Top-Down technology and delay perception is proposed in this article. Firstly, the tree structure thought is introduced therein to divide 
WSN sensor nodes into multiple single-layer clusters with different sizes so as to effectively reduce the delay; secondly, Top-Down technology is adopted to design the network structure formation algorithm, thus to optimize the energy consumption of the proposed data collection mechanism through the communication distance minimization; finally, the delay and the energy consumption of the proposed data collection method are verified by relevant experiment.

\section{Design of the Proposed Network Structure}

In order to avoid the sharp increase of data collection time, the tree structure thought [8] is introduced in this article to design a new network structure. Due to the poor reliability of the traditional tree structure, in order to improve the reliability of the proposed network structure, the improved fat tree structure with good stability is directly adopted as follows (please refer to literature [9]): define and divide the intra-domains in the traditional fat tree structure, increase some intra-domain interconnection nodes for interconnection through the nodes at the 3rd layer of each intra-domain in order to finally generate the intra-domain interconnection structure, and improve the fat tree structure through the intra-domain interconnection structure in order to improve the connectivity among the tree nodes and the fault-tolerant capability. Meanwhile, in order to ensure that the proposed network structure can provide maximum data collection efficiency, the total node number of the proposed WSN network structure is set as $\mathrm{N}=2^{\mathrm{p}}, \mathrm{p}=1,2, \ldots$. In such network structure, WSN sensor nodes are divided into multiple single-layer clusters with different sizes in order to enable the cluster heads to communicate with the data fusion center in an interlaced mode. In such structure, if WSN has N nodes, then these nodes are divided into K clusters:

$$
\begin{aligned}
& \sum_{\mathrm{i}=1}^{\mathrm{K}-1} \mathrm{~m}_{\mathrm{i}}<\mathrm{N}<\sum_{\mathrm{i}=1}^{\mathrm{K}} \mathrm{m}_{\mathrm{i}} \\
& \mathrm{m}_{\mathrm{i}}=2 \mathrm{~m}_{\mathrm{i}-1}, \mathrm{~m}_{\mathrm{i}}=1
\end{aligned}
$$

In the above formula, $\mathrm{m}_{\mathrm{i}}$ is the maximum node number in the $\mathrm{i}$ th cluster; $\mathrm{K}$ is the cluster number.

Model (2) is simplified as follows:

$$
\mathrm{m}_{\mathrm{i}}=2^{\mathrm{i}-1}
$$

According to Models (1) and (3), the following formula can be obtained:

$$
2^{\mathrm{K}-1}-1<\mathrm{N} \leq 2^{\mathrm{K}}-1
$$

In the proposed network structure, each cluster member obtains a rank ( $\operatorname{Rank} \in[1, \mathrm{p}]$ ), and the node with the rank as $\mathrm{T}$ can generate $\mathrm{T}-1$ data chains, and each data chain has T-1 nodes, wherein these $\mathrm{T}-1$ nodes have different ranks $1,2, \ldots . . \mathrm{T}-1$ and become the child nodes of the node with the rank as T. Meanwhile, the node with the rank as T generates a data chain to make the nodes in the chain have higher ranks, and such nodes with higher ranks will become the father nodes. For example, the cluster head is the node with highest rank in the whole WSN network. In the designed network structure, the cluster head can generate the data chain for communicating with the base station rather than generate the data chain able to form the nodes with higher ranks. According to the above logic, the node ranks are as shown in Table 1.

Table 1. Cluster Member Distribution in the Proposed Network Structure

\begin{tabular}{ccccccc}
\hline Rank & 1 & 2 & 3 & $\ldots \ldots$ & $\log _{2} \mathrm{~N}-1$ & $\log _{2} \mathrm{~N}$ \\
\hline Node Quantity & $\mathrm{N} / 2$ & $\mathrm{~N} / 2^{2}$ & $\mathrm{~N} / 2^{3}$ & $\ldots \ldots$ & $\mathrm{N} / 2^{\log _{2} \mathrm{~N}-1}$ & $\mathrm{~N} / 2^{\log _{2} \mathrm{~N}}$ \\
\hline
\end{tabular}


During data collection, the Kth cluster in the proposed network structure is adopted to connect the data fusion center. If the cluster is smaller, then the data collection process is shorter. Therefore, before the Kth cluster is fully filled, the first (K-1) clusters have been fully filled with the cluster members. For example, if $\mathrm{N}$ is set as $\mathrm{N}=16$, then the designed network structure model is as shown in Figure 1. According to Figure 1, if the whole time domain is divided into continuous time slots, then the model needs 5 time slots.

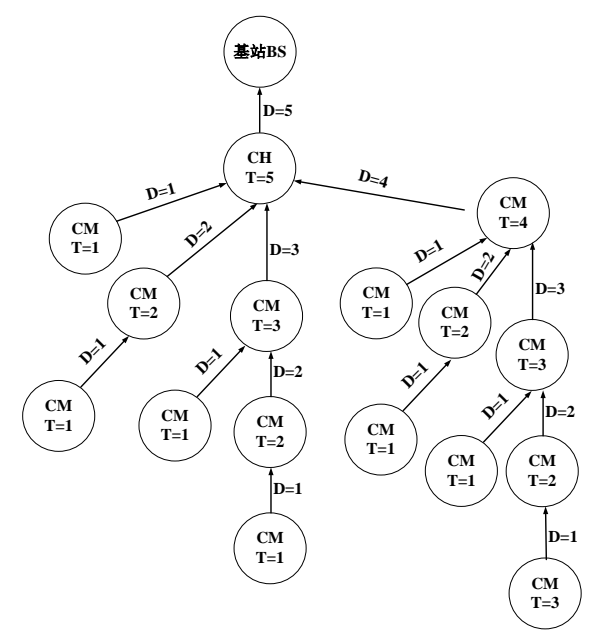

\section{Figure 1. The Proposed Network Structure (Node number N=16) (BS: base station; CM: cluster member; $\mathrm{CH}$ : cluster head; T: node rank; D: continuous rank; Arrow: data chain)}

Lemma 1: For a network with $\mathrm{N}$ nodes $\left(\mathrm{N}=2^{\mathrm{p}}, \mathrm{p}=1,2, \ldots\right)$, if the data packets collected by the sensors thereof are highly correlated with each other, then the nodes can integrate all data packets received thereby through data/decision fusion technology into a single data packet. Although the proposed network structure is adopted, node $i$ with the rank as $\mathrm{T} \geq 2$ needs (T-1) time slots to collect the output data of all child nodes.

Proof: Since the network structure includes $\mathrm{N}$ nodes $\left(\mathrm{N}=2^{\mathrm{p}}, \mathrm{p}=1,2, \ldots\right)$, the time slot for the node with the rank as $\mathrm{T}=2$ to collect the data from all child nodes is equal to the child node quantity, namely $\mathrm{D}=1$; obviously, when $\mathrm{T}=2$, the above lemma is true. For node $\mathrm{i}$ with the rank as $\mathrm{T}=\mathrm{n}+1$, it has $\mathrm{n}$ child nodes directly connected thereto and each node has different ranks from 1 to $n$, so these nodes need $(0 \sim \mathrm{n}-1)$ time slots to collect the data of all child nodes and meanwhile need an additional time slot to broadcast the collected data to node i; obviously, the maximum time slot for node i to collect the output data of all child nodes thereof is $\mathrm{T}-1=\mathrm{n}$., q.e.d.

Theorem 1: For a network with $\mathrm{N}$ nodes $\left(\mathrm{N}=2^{\mathrm{p}}, \mathrm{p}=1,2, \ldots\right)$, if the data packets collected by the sensors thereof are highly correlated with each other, then the nodes can integrate all data packets received thereby through data/decision fusion technology into a single data packet. For the designed network structure, the time slot $t(N)$ for the base station to collect data from the whole network is as follows:

$$
\mathrm{t}(\mathrm{N})=\log _{2} \mathrm{~N}+1
$$

Proof: For a network with $\mathrm{N}$ nodes $\left(\mathrm{N}=2^{\mathrm{p}}, \mathrm{p}=1,2, \ldots\right)$, according to the proposed network structure, the cluster head is the node with the highest rank:

$$
\mathrm{T}_{\max }=\log _{2} \mathrm{~N}+1
$$

Then, according to Lemma 1, the time slot for the cluster head with the rank as $\mathrm{T}_{\max }$ to collect the data from all child nodes is as follows:

$$
\mathrm{t}(\mathrm{N})=\mathrm{T}_{\max }-1=\log _{2} \mathrm{~N}
$$


Therefore, the number of the time slots for the base station to collect the data from the network is equal to that for the cluster head to collect the data from all child nodes:

$$
t(N)=\log _{2} N+1
$$

According to Formula (8) and Figure 1, the proposed network structure is an optimized tree structure: (1) each sensor node can communicate with one node only for one time; (2) the data fusion can be achieved at each node; (3) the network is composed of multiple single-layer clusters.

\section{Modeling}

\subsection{Delay during Data Collection}

In WSN, the sample data collected by different nodes are highly correlated with each other. Data fusion is achieved during data collection, for example: two data packets with the sizes respectively as $\alpha_{\mathrm{i}}, \alpha_{\mathrm{j}}$ can be fused into a single data packet with the size as $\alpha_{k}=r\left(\alpha_{i}+\alpha_{j}\right) \leq\left(\alpha_{i}+\alpha_{j}\right), r \in[0.5,1]$, wherein $r$ is the compression ratio. For a network with the sensor node number as $2^{\mathrm{K}-1}-1<\mathrm{N}<2^{\mathrm{K}}-1$, according to the proposed network structure, these nodes are divided into $\mathrm{K}$ clusters, wherein the first $\mathrm{K}-1$ clusters are fully filled with the cluster members $\mathrm{CM}$ and need $\left(\sum_{\mathrm{i}=1}^{\mathrm{K}-1} \mathrm{~m}_{\mathrm{i}}=2^{\mathrm{K}-1}-1\right)$ time slots to broadcast the data thereof to the fusion center, and the Kth cluster is fully filled with $\left(\mathrm{N}-2^{\mathrm{K}-1}-1\right)$ cluster members $\mathrm{CM}$. At this moment, the cluster head $\mathrm{CH}$ needs $\left(\mathrm{N}-2^{\mathrm{K}-1}+1\right)$ time slots to transmit the data to the fusion center. Therefore, the delay $\mathrm{D}(\mathrm{N})$ during data collection in the proposed network structure is as follows:

$$
\begin{aligned}
\mathrm{D}(\mathrm{N}) & =\mathrm{Z}^{\mathrm{K}-1}-1+\mathrm{N}-\mathrm{Z}^{\mathrm{K}-1}+1 \\
= & \mathrm{N}=\mathrm{D}(\mathrm{N})_{\text {max }}
\end{aligned}
$$

Generally speaking, $\mathrm{D}(\mathrm{N})$ depends on the compression ratio $\mathrm{r}$ and the size of the Kth cluster. When the (K-1)th cluster finishes the communication, the Kth cluster can communicate with the fusion center. Then, $\mathrm{D}(\mathrm{N})$ is as follows:

$$
\begin{aligned}
& \mathrm{D}(\mathrm{N})=\left\lceil\max \left\{\mathrm{T}^{\prime}\left(2^{\mathrm{K}-1}-1\right), \mathrm{N}-2^{\mathrm{K}-1}-1\right\}\right\rceil \\
&+\left\lceil\mathrm{r}^{\mathrm{N}-2^{\mathrm{K}-1}-1}+\sum_{\mathrm{j}=1}^{\mathrm{N}-2^{\mathrm{K}-1}} \mathrm{r}^{\mathrm{j}}\right\rceil \\
& \mathrm{T}^{\prime}\left(2^{\mathrm{K}-1}-1\right)=\mathrm{T}^{\prime}\left(\mathrm{m}_{\mathrm{K}-1}-1\right) \\
&=\mathrm{m}_{\mathrm{K}-1}-1+\left\lceil\mathrm{r}^{\mathrm{m}-1-1}+\sum_{\mathrm{j}=1}^{\mathrm{m}_{\mathrm{K}-1}-1} \mathrm{r}^{\mathrm{j}}\right]
\end{aligned}
$$

In the above formula, $\lceil\mathrm{x}\rceil$ denotes the minimum integer more than $\mathrm{x} ; \mathrm{T}^{\prime}\left(2^{\mathrm{K}-1}-1\right)$ denotes the delay needed by the first K-1 clusters during data collection.

\subsection{Energy Consumption}

The energy consumption of WSN nodes is mainly incurred by the receiving and dispatching modules thereof. WSN node can be regarded as a device composed of three main units, namely: micro-programmed control unit (MCU), transceiver unit (TCR) and sensor circuit board unit (SB). The energy consumption model E of WSN network structure is as follows:

$$
\mathrm{E}_{\mathrm{i}_{\_} \mathrm{SN}}=\mathrm{E}_{\mathrm{i}_{\_} \mathrm{MCU}}+\mathrm{E}_{\mathrm{i}_{\_} \mathrm{TCR}}+\mathrm{E}_{\mathrm{i}_{\_} \mathrm{SB}}
$$




$$
\mathrm{E}_{\mathrm{i}_{-} \mathrm{TCR}}=\mathrm{E}_{\mathrm{i}_{-} \mathrm{TCR}_{-} \mathrm{RX}}+\mathrm{E}_{\mathrm{i}_{-} \mathrm{TCR}_{-} \mathrm{TX}}\left(\mathrm{d}_{\mathrm{i}}\right)
$$

In the above formula, $\mathrm{E}_{\mathrm{i}_{-} \mathrm{MCU}}$ denotes the energy consumption of MCU; $\mathrm{E}_{\mathrm{i}_{-} \text {TCR }}$ denotes the energy consumption of TCR ; $\mathrm{E}_{\mathrm{i}_{\perp} \mathrm{SB}}$ denotes the energy consumption of $\mathrm{SB} ; \mathrm{E}_{\mathrm{i}_{-} \mathrm{TCR}-\mathrm{RX}}$ denotes the energy consumption of TCR for data receiving; $E_{\mathrm{i}_{-} \text {TCR }-T X}\left(d_{i}\right)$ denotes the energy consumption of TCR at the transmission distance of $d_{i}$.

Since the proposed network structure includes $\mathrm{N}$ nodes $\left(2^{\mathrm{K}-1}-1<\mathrm{N}<2^{\mathrm{K}}-1\right)$, the total energy consumption $\mathrm{E}_{\text {тот }}(\mathrm{N})$ of the network is as follows:

$$
\mathrm{E}_{\text {TOT }}(\mathrm{N})=\sum_{\mathrm{i}=1}^{\mathrm{N}}\left(\mathrm{E}_{\mathrm{i}_{-} \mathrm{MCU}}+\mathrm{E}_{\mathrm{i}_{-} \mathrm{TCR}_{-} \mathrm{RX}}+\mathrm{E}_{\mathrm{i}_{-} \mathrm{TCR}_{-} \mathrm{TX}}\left(\mathrm{d}_{\mathrm{i}}\right)+\mathrm{E}_{\mathrm{i}_{-} \mathrm{SB}}\right)
$$

Model (14) is simplified to obtain the following model:

$$
\mathrm{E}_{\text {TOT }}(\mathrm{N})=\mathrm{C}_{1}+\sum_{\mathrm{i}=1}^{\mathrm{N}} \mathrm{E}_{\mathrm{i}_{-} \mathrm{TCR}_{-} \mathrm{TX}}\left(\mathrm{d}_{\mathrm{i}}\right)
$$

In the above formula, $\mathrm{C}_{1}$ is a constant; $\mathrm{d}_{\mathrm{i}}$ denotes communication distance.

If the path loss index is 2 , then $\mathrm{E}_{\mathrm{i}_{-} \text {TCR_TX }}\left(\mathrm{d}_{\mathrm{i}}\right)$ is as follows:

$$
\mathrm{E}_{\mathrm{i}_{-} \mathrm{TCR}_{-} \mathrm{TX}}\left(\mathrm{d}_{\mathrm{i}}\right)=\mathrm{E}_{\mathrm{i}_{-} \mathrm{TCR}_{-} \mathrm{EC}}+\mathrm{E}_{\mathrm{i}_{-} \mathrm{TCR}_{-} \mathrm{PA}} \mathrm{d}_{\mathrm{i}}^{2}
$$

In the above formula, $\mathrm{E}_{\mathrm{i}_{-} \mathrm{TCR}_{-} \mathrm{EC}}$ denotes the circuit energy consumption of TCR ; $\mathrm{E}_{\mathrm{i}_{-} \text {TCR_PA }}$ denotes the power amplification energy consumption of TCR .

Since $\mathrm{E}_{\mathrm{i}_{-} \mathrm{TCR} \mathrm{R}_{-} \mathrm{EC}}$ and $\mathrm{E}_{\mathrm{i}_{-} \mathrm{TCR} \mathrm{R}_{-} \mathrm{PA}}$ are constants, Model (15) can be converted into the following model:

$$
\mathrm{E}_{\mathrm{TOT}}(\mathrm{N})=\mathrm{C}_{1}+\mathrm{C}_{2}+\mathrm{C}_{3} \sum_{\mathrm{i}=1}^{\mathrm{N}} \mathrm{d}_{\mathrm{i}}^{2}
$$

In the above formula, $\mathrm{C}_{1}, \mathrm{C}_{2}, \mathrm{C}_{3}$ are all constants.

According to Model (17), the energy consumption of the proposed network structure can be minimized through $\sum_{\mathrm{i}=1}^{\mathrm{N}} \mathrm{d}_{\mathrm{i}}{ }^{2}$ reduction. Hereto, Top-Down technology is introduced in this article to design the network structure formation algorithm, thus to optimize the energy consumption of the proposed data collection mechanism through the communication distance minimization.

\section{Network Structure Formation Algorithm}

Top-Down technology is a centralized control algorithm [10]. According to this technology, if the base station BS is assumed to have the coordinate information of all sensors in WSN, then the base station will indicate the sensor nodes to establish necessary data chains and form the corresponding network structure. For a network with $\mathrm{N}$ nodes $\left(\mathrm{N}=2^{\mathrm{p}}, \mathrm{p}=2,3, \ldots\right)$, the specific steps are as follows:

(1) Firstly, regard the whole network as a network in full connection, wherein the connection refers to the data chain established between two wireless sensor nodes for transmitting data packets, and if two nodes are not directly connected to form a data chain, such connection is defined as discontinuous connection; the connection degree of the wireless sensor nodes is used to display the total number of the data chains related to these nodes; if the connection degree of a node is 3 , then it indicates that this node will form the corresponding data chains to connect other three nodes. For the proposed network structure with $\mathrm{N}$ nodes $\left(\mathrm{N}=2^{\mathrm{p}}, \mathrm{p}=2,3, \ldots\right)$, set the connection degree of each node as $\mathrm{N}-1$, set the corresponding node set as $\mathrm{H}_{\mathrm{S}=1}^{\prime}$, and set $\mathrm{b}=\mathrm{N} / 2$. 
(2) Select $b$ nodes from set $\mathrm{H}_{\mathrm{S}}^{\prime}$ to form set $\mathrm{H}_{\mathrm{S}+1}$ and minimize $\sum_{\mathrm{i}, \mathrm{j} \in \mathrm{H}_{\mathrm{s}+1}} \mathrm{~d}_{\mathrm{i}, \mathrm{j}}{ }^{2}$, wherein $\mathrm{d}_{\mathrm{i}, \mathrm{j}}{ }^{2}$ denotes the transmission distance between nodes $\mathrm{i}, \mathrm{j}$, and the remaining nodes in $\mathrm{H}_{\mathrm{S}=1}^{\prime}$ form set $\mathrm{H}_{\mathrm{S}+1}$, and the algorithm will eliminate all data chains among these nodes; then, set iteration $\mathrm{S} \leftarrow \mathrm{S}+1, \mathrm{~b} \leftarrow \mathrm{b} / 2$.

(3) Repeat step (2) till $\mathrm{b}<2$; then, set $g=2$.

(4) If the connection degree of the nodes is $\mathrm{N}-\mathrm{g}$, then form set $\mathrm{L}$; if the connection degree is more than $\mathrm{N}-\mathrm{g}$, then form set $\mathrm{U}$; enable the two sets $\mathrm{L}$ and $\mathrm{U}$ to have the same number of nodes; if each node in set $\mathrm{L}$ is only connected to a single node in set $\mathrm{U}$, then the data chains among the nodes in the two sets will be reduced; if the data chains are reduced, then set $\mathrm{g} \leftarrow \mathrm{g} \times 2$.

(5) Repeat step (2) till $g=\mathrm{N}$.

According to Figure 1, the proposed network structure is a tree structure. In such structure, a child node is only connected to a father node. The edges among the nodes with the same connection degree will be eliminated through steps (1) and (2). Therefore, according to the output result of step (2), a child node can be connected to multiple father nodes. The surplus edges of the child nodes will be eliminated through steps (3) and (4) in order to ensure the minimization of the total weight of the remaining edges. After step (4), a child node is only connected to a father node.

In order to specifically explain the formation process of the proposed network structure based on Top-Down technology, the network with $\mathrm{N}$ nodes $(\mathrm{N}=8)$ is taken as an example in this article.

(a) For the WSN network with $\mathrm{N}$ nodes $(\mathrm{N}=8)$, set the connection degree of each node as $\mathrm{N}-1=7$ (because the node number is 8 ), set the set formed by 8 nodes as $\mathrm{H}_{\mathrm{S}=1}^{\prime}$, and define the parameter $\mathrm{b}=\mathrm{N} / 2=4$, as shown in Figure 2 (i).

(b) Select 4 nodes from set $\mathrm{H}_{\mathrm{S}=1}^{\prime}$ to form set $\mathrm{H}_{\mathrm{S}+1}\left(\mathrm{H}_{2}\right)$ and minimize the total edge weight, adopt the dynamic planning technology [11] to solve such combination problem, wherein since the total edge weight of the four nodes $\mathrm{C}, \mathrm{D}, \mathrm{E}$ and $\mathrm{F}$ is the highest as shown in Figure 3 (ii), the set $\mathrm{H}_{2}$ is composed of the four nodes $\mathrm{C}, \mathrm{D}, \mathrm{E}$ and F; then, eliminate the connection among the nodes, as shown in Figure 2(ii); then, set $b=b / 2=2$ and $S=S+1=2$.

(c) Since $b=2 \geq 2$, return to step (2) for continuous execution; select 2 nodes from set $\mathrm{H}_{2}$ to form set $\mathrm{H}_{3}$ and minimize the total weight of the nodes in $\mathrm{H}_{3}$, wherein since the total weight of nodes $\mathrm{A}$ and $\mathrm{H}$ or nodes $\mathrm{G}$ and $\mathrm{B}$ is the highest, randomly select an optional pair of the nodes from the two pairs to form set $\mathrm{H}_{3}$; as shown in Figure 2(iii), select nodes $A$ and $\mathrm{H}$ to form set $\mathrm{H}_{3}$, and select the remaining nodes $\mathrm{B}$ and $\mathrm{G}$ to form $\operatorname{set} \mathrm{H}_{3}^{\prime}$; then, eliminate the connection among the nodes, as shown in Figure 2 (iii); then, set $b=b / 2=1$ and $S=S+1=3$.

(d) Since $b=1<2$, continuously execute the algorithm and set $g=2$; if the connection degree of the nodes is $\mathrm{N}-\mathrm{g}=6$ (as the connection degree of nodes $\mathrm{A}$ and $\mathrm{H}$ shown in the figure), then form set $\mathrm{L}$; if the connection degree is more than $\mathrm{N}-\mathrm{g}$ (as the connection degree of nodes $\mathrm{B}$ and $\mathrm{G}$ shown in the figure), then form set $\mathrm{U}$; gradually reduce the node connections between set $\mathrm{L}$ and set $\mathrm{U}$ till each node in set $\mathrm{L}$ is only connected to one node in set $U$ in order to minimize the total edge weight, as shown in Figure 2(iv); then, adopt Munkres allocation algorithm[12] to solve the weight matching problem; then, set $\mathrm{g} \rightarrow 2 \mathrm{~g}=4$.

(e) Since $g=4<N$, continuously execute the above steps; if the connection degree of the nodes is $\mathrm{N}-\mathrm{g}=4$ (as the connection degree of nodes $\mathrm{C}, \mathrm{D}, \mathrm{E}$ and $\mathrm{F}$ shown in Figure 
$(\mathrm{V})$, then form set $\mathrm{L}$; if the connection degree is more than $\mathrm{N}-\mathrm{g}$ (as the connection degree of nodes A, B, G and $\mathrm{H}$ shown in Figure (V)), then form set $\mathrm{U}$; gradually reduce the node connections between set $\mathrm{L}$ and set $\mathrm{U}$ till each node in set $\mathrm{L}$ is only connected to one node in set $\mathrm{U}$ in order to minimize the total edge weight, as shown in Figure $2(\mathrm{~V})$; then, set $\mathrm{g} \rightarrow 2 \mathrm{~g}=8$.

(f) When $\mathrm{g}=8=\mathrm{N}$, the algorithm is ended. The network formed at this moment is composed of the mutually connected nodes with the connection degree as $\log _{2} \mathrm{~N}=3$, wherein one of the two nodes, near to the base station (as node B shown in Figure (Vi)), will be selected as the cluster head and will be directly connected to the base station. Meanwhile, the connection degree of the cluster head is $\log _{2} N+1=4$.

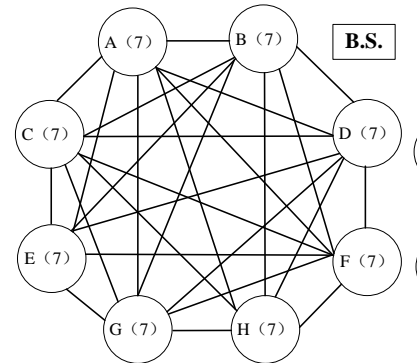

(i)

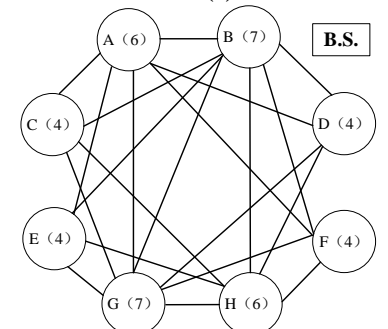

(iii)

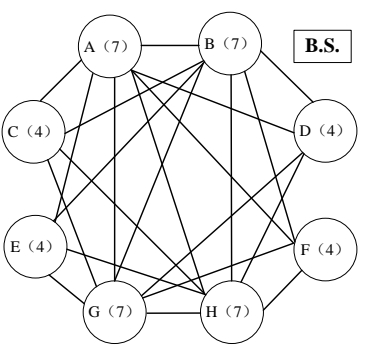

(ii)

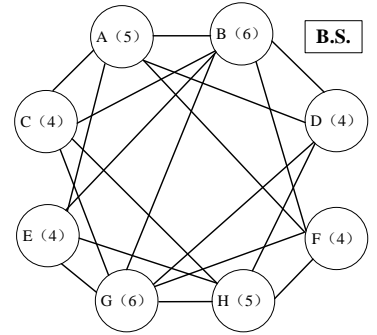

(iV)

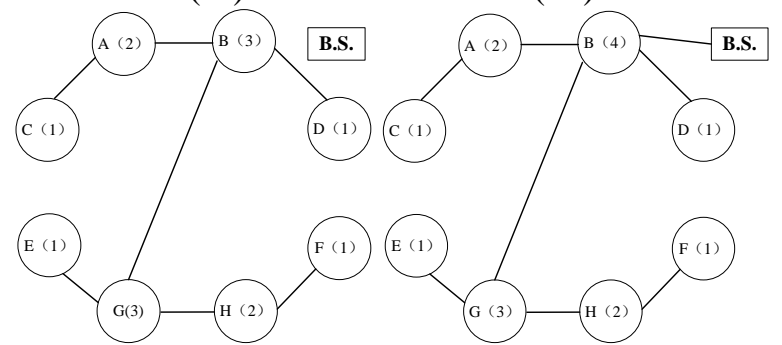

(V)

Figure 2. Network Structure Formation $(\mathrm{N}=8)$ (Circle: sensor nodes; Rectangle: base station)

\section{Energy Consumption Optimization of the Proposed Network Structure}

In order to optimize the energy consumption of the proposed network structure and maintain the energy consumption of the data collection at a minimum level, it is necessary to minimize the communication distance of the network structure. Specifically, the optimization steps are as follows:

(1) For a network with $\mathrm{N}$ nodes $\left(2^{\mathrm{K}-1}-1<\mathrm{N}<2^{\mathrm{K}}-1\right)$, the nodes are classified by a descending order according to the rest energy $E_{\text {res }}$ of the nodes to form set $S=\left\{s_{1}, s_{2}, \ldots . . s_{N}\right\}$, namely $\mathrm{E}_{\text {res }}\left(\mathrm{s}_{1}\right) \geq \mathrm{E}_{\mathrm{res}}\left(\mathrm{s}_{2}\right) \ldots \ldots . . \mathrm{E}_{\mathrm{res}}\left(\mathrm{s}_{\mathrm{N}}\right)$. 
(2) Select the first $\mathrm{k}$ elements in set $\mathrm{S}$ and regard them as the cluster heads of the network.

(3) Adopt the binary integer planning method[13] to minimize the communication distance between the cluster head and the cluster member:

$$
\begin{gathered}
\min \sum_{\mathrm{i}=1}^{\mathrm{K}} \sum_{\mathrm{j}=\mathrm{K}+1}^{\mathrm{N}} \mathrm{x}_{\mathrm{ij}} \mathrm{d}_{\mathrm{ij}}{ }^{2} \\
\sum_{\mathrm{j}=1}^{\mathrm{K}} \mathrm{x}_{\mathrm{ij}}, \forall \mathrm{i} \in[\mathrm{K}+1, \ldots \ldots . \mathrm{N}] \\
\sum_{\mathrm{i}=\mathrm{K}+1}^{\mathrm{N}} \mathrm{x}_{\mathrm{ij}}=\mathrm{m}_{\mathrm{K}-\mathrm{j}+1}-1, \forall \mathrm{j} \in[2, \ldots \ldots . \mathrm{K}-1] \\
\sum_{\mathrm{i}=\mathrm{K}+1}^{\mathrm{N}} \mathrm{x}_{\mathrm{ij}} \leq \mathrm{m}_{\mathrm{K}}
\end{gathered}
$$

In the above formula, $\mathrm{d}_{\mathrm{ij}}$ denotes the distance between nodes $\mathrm{s}_{\mathrm{i}}, \mathrm{s}_{\mathrm{j}}, \mathrm{x}_{\mathrm{ij}}$ is used to display the coordinate of the connection between nodes $\mathrm{s}_{\mathrm{i}}, \mathrm{s}_{\mathrm{j}} ; \mathrm{m}_{\mathrm{K}}$ denotes the maximum number of the nodes in the $\mathrm{K}$ th cluster.

Specifically, the cluster head is mainly responsible for collecting the data from the cluster members, and the cluster member is responsible for data fusion and for broadcasting the information to the fusion center, wherein the energy consumption of the cluster head is the highest. Therefore, the node with the highest rest energy is regarded as the cluster head in step (2), and the communication distance between the cluster head and the cluster member is minimized in step (3). Additionally, Model (19) is adopted to ensure that a cluster member is only connected to a cluster head, Models (20) and (21) are adopted to ensure that the $\mathrm{K}$ th cluster is fully filled with the cluster members.

\section{Simulation Experimental and Analysis}

The delay and the energy consumption of the proposed network structure are tested in Matlab tool. In order to present the superiority of the proposed network structure, existing data collection network structures with good performance are taken as the control group: literature [14] ---- network structure based on minimum spanning tree MST, literature [15] ---- CTP based network structure. In the experiment, $\mathrm{N}$ nodes $(\mathrm{N} \in[2,98])$ are randomly distributed in a sensing area with the size as $100 \times 100 \mathrm{~m}^{2}$, wherein the center of the sensing scope is $(50 \mathrm{~m}, 50 \mathrm{~m})$. The specific parameters are as shown in Table 2.

Table 2. Parameter Setting of Simulation Experiment

\begin{tabular}{cc}
\hline Name & Measurement \\
\hline $\mathrm{E}_{\mathrm{i}_{\_} \mathrm{TCR} \_\mathrm{RX}}$ & $60 \times 10^{-6} \mathrm{~J} / \mathrm{bit}$ \\
$\mathrm{E}_{\mathrm{i}_{-} \mathrm{TCR} \_\mathrm{EC}}$ & $60 \times 10^{-6} \mathrm{~J} / \mathrm{bit}$ \\
$\mathrm{E}_{\mathrm{i}_{-} \mathrm{TCR} \_\mathrm{PA}}$ & $150 \times 10^{-9} \mathrm{~J} / \mathrm{bit}$ \\
$\mathrm{E}_{\mathrm{i}_{-} \mathrm{MCU}}$ & $5 \times 10^{-6} \mathrm{~J} / \mathrm{bit}$ \\
$\mathrm{E}_{\mathrm{i}_{-} \mathrm{SB}}$ & $100 \times 10^{-6} \mathrm{~J} / \mathrm{bit}$ \\
Data Packet Size & $1024(\mathrm{bits})$ \\
Data Rate & $64(\mathrm{kbps})$ \\
Power Voltage & $3(\mathrm{~V})$ \\
Data Compression Ratio & 0.5 \\
\hline
\end{tabular}




\subsection{Delay during Data Collection}

Figure 3 shows the delay test results of the proposed network structure for data collection and the control group. According to the figure, when the node number is increased, the delay of these WSN data collection technologies is also increased. Therein, the delay of the proposed mechanism during data collection is minimum and has a relatively small increase range, and when the node number is more than 50, the proposed mechanism has relatively stable state, because the tree structure thought is adopted in this article to divide WSN sensor nodes into multiple single-layer clusters with different sizes so as to optimize the communication distance among the nodes and enable the cluster head to complete data transmission and communication in an interlaced mode. Although the tree structure thought is also adopted in literatures [14] and [15] and the communication distance is also minimized to reduce the energy consumption of the network, yet large delay is still caused in these network structures due to the division of the nodes into multilayer structures.

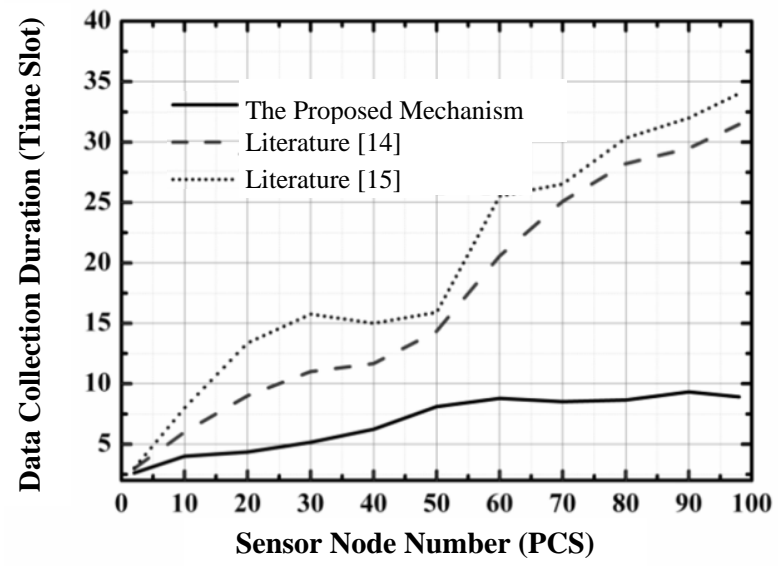

Figure 3. Delay Tests of Different Network Structures for Data Collection

\subsection{Energy Consumption}

Figure 4 shows the energy consumption test results of the proposed network structure for data collection and the control group. According to the figure, the energy consumption of the network structure proposed in literature [14] is approximate to that of the proposed network structure, but the energy consumption of the network structure proposed in literature [15] is slightly more than that of the former two, because Top-Down technology is adopted in the proposed algorithm to design the formation algorithm of the proposed network structure so as to minimize $\sum_{\mathrm{i}=1}^{\mathrm{N}} \mathrm{d}_{\mathrm{i}}{ }^{2}$, and meanwhile the binary integer planning method is also introduced therein to minimize the communication distance so as to maintain the energy consumption of the proposed network structure at a relatively low level. 


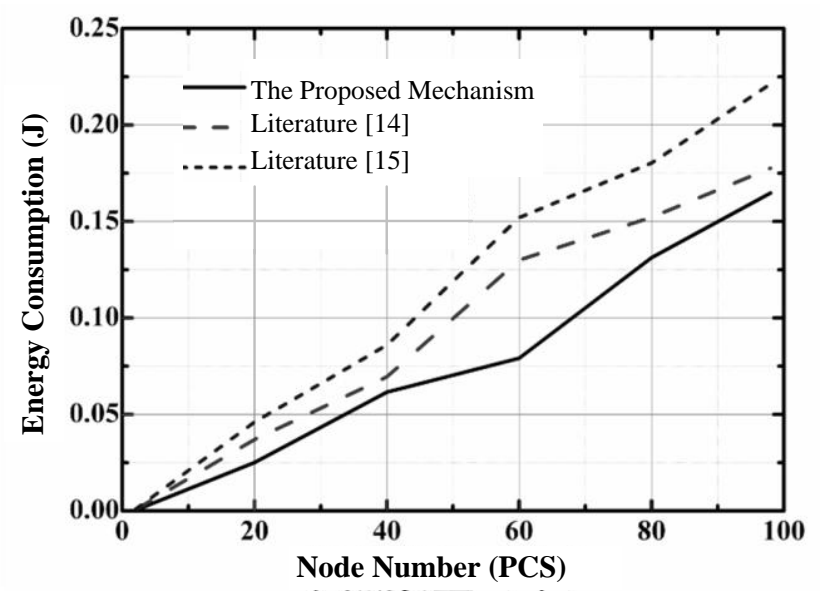

Figure 4. Energy Consumption Tests of Different Network Structures for Data Collection

\subsection{Network Life Cycle}

Figure 5 shows the delay test results of the proposed network structure for data collection and the control group. According to the figure, when the node number is increased, the network survival time of the three network structures is reduced and the corresponding energy consumption is increased. Specifically, the network structure proposed in literature [15] has poorer network performance and shorter life cycle, and the proposed network structure has longest survival time. According to Figure 5, the proposed network structure can maintain the energy consumption at a relatively low level, thus prolonging the network survival time; the network structure proposed in literature [15] has largest energy consumption and accordingly has the shortest life cycle.

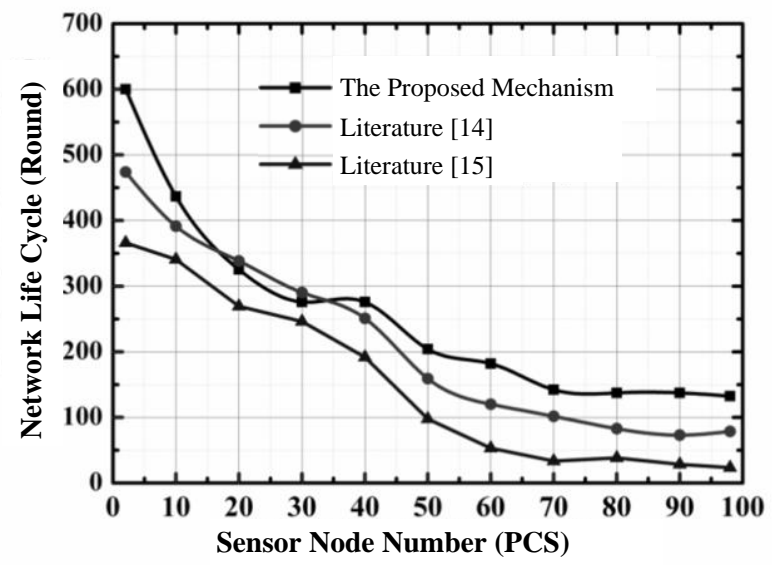

Figure 5. Life Cycle Tests of Different Network Structures for Data Collection

\section{Conclusion}

In order to reduce the delay during data collection and optimize the energy consumption of the network, the tree structure thought is introduced in this article. Meanwhile, WSN sensor nodes are divided into multiple single-layer clusters with different sizes to construct a new network structure and enable the cluster head to complete the data communication in an interlaced mode, thus significantly reducing the delay during data collection; afterwards, the model for calculating the delay and the energy consumption of WSN data collection is 
established in this article; then, Top-Down technology is adopted in this article to design the formation algorithm of the proposed network structure in order to optimize the energy consumption of the proposed data collection mechanism through the communication distance minimization. The experiment result shows: the proposed network structure can effectively reduce the delay during WSN data collection and maintain the whole communication energy consumption at a relatively low level.

\section{Acknowledgement}

The paper is supported by the project "Research on the key technology of load balancing in wireless Mesh network gateway No. 61103202"

\section{References}

[1] J. Hu and Z. Gao, "Distinction immune genes of hepatitis-induced heptatocellular carcinoma", Bioinformatics, vol. 28, no. 24, (2012), pp. 3191-3194.

[2] J. Yang, B. Chen and J. Zhou, "A Low-Power and Portable Biomedical Device for Respiratory Monitoring with a Stable Power Source", Sensors, vol. 15, no. 8, (2015), pp. 19618-19632.

[3] G. Bao, L. Mi, Y. Geng and K. Pahlavan, "A computer vision based speed estimation technique for localizing the wireless capsule endoscope inside small intestine", 36th Annual International Conference of the IEEE Engineering in Medicine and Biology Society (EMBC), (2014).

[4] X. Song and Y. Geng, "Distributed community detection optimization algorithm for complex networks", Journal of Networks, vol. 9, no. 10, (2014), pp. 2758-2765.

[5] D. Jiang, X. Ying and Y. Han, "Collaborative multi-hop routing in cognitive wireless networks", Wireless Personal Communications, (2015), pp. 1-23.

[6] Z. Lv, A. Halawani and S. Feng, "Multimodal hand and foot gesture interaction for handheld devices", ACM Transactions on Multimedia Computing, Communications, and Applications (TOMM), vol. 11, no. 1s, (2014), p. 10.

[7] G. Liu, Y. Geng and K. Pahlavan, "Effects of calibration RFID tags on performance of inertial navigation in indoor environment", 2015 International Conference on Computing, Networking and Communications (ICNC), (2015).

[8] J. He, Y. Geng, Y. Wan, S. Li and K. Pahlavan, "A cyber physical test-bed for virtualization of RF access environment for body sensor network", IEEE Sensor Journal, vol. 13, no. 10, (2013), pp. 3826-3836.

[9] W. Huang and Y. Geng, "Identification Method of Attack Path Based on Immune Intrusion Detection", Journal of Networks, vol. 9, no. 4, (2014), pp. 964-971.

[10] X. Li, Z. Lv and J. Hu, "XEarth: A 3D GIS Platform for managing massive city information", Computational Intelligence and Virtual Environments for Measurement Systems and Applications (CIVEMSA), 2015 IEEE International Conference on IEEE, (2015), pp. 1-6.

[11] J. He, Y. Geng, F. Liu and C. Xu, "CC-KF: Enhanced TOA Performance in Multipath and NLOS Indoor Extreme Environment”, IEEE Sensor Journal, vol. 14, no. 11, (2014), pp. 3766-3774.

[12] N. Lu, C. Lu, Z. Yang and Y. Geng, "Modeling Framework for Mining Lifecycle Management", Journal of Networks, vol. 9, no. 3, (2014), pp. 719-725.

[13] Y. Geng and K. Pahlavan, "On the accuracy of rf and image processing based hybrid localization for wireless capsule endoscopy", IEEE Wireless Communications and Networking Conference (WCNC), (2015).

[14] X. Li, Z. Lv and J. Hu, "Traffic management and forecasting system based on 3d gis", Cluster, Cloud and Grid Computing (CCGrid), 2015 15th IEEE/ACM International Symposium on, (2015), pp. 991-998.

[15] S. Zhang and H. Jing, "Fast log-Gabor-based nonlocal means image denoising methods", Image Processing (ICIP), 2014 IEEE International Conference on IEEE, (2014), pp. 2724-2728.

[16] D. Jiang, Z. Xu and Z. Chen, "Joint time-frequency sparse estimation of large-scale network traffic", Computer Networks, vol. 55, no. 15, (2011), pp. 3533-3547.

[17] J. Hu, Z. Gao and W. Pan, "Multiangle Social Network Recommendation Algorithms and Similarity Network Evaluation", Journal of Applied Mathematics, (2013).

[18] J. Hu and Z. Gao, "Modules identification in gene positive networks of hepatocellular carcinoma using Pearson agglomerative method and Pearson cohesion coupling modularity", Journal of Applied Mathematics, (2012).

[19] Z. Lv, A. Tek and F. Da Silva, "Game on, science-how video game technology may help biologists tackle visualization challenges", PloS one, vol. 8, no. 3, (2013), p. 57990.

[20] T. Su, W. Wang and Z. Lv, "Rapid Delaunay triangulation for randomly distributed point cloud data using adaptive Hilbert curve", Computers \& Graphics, vol. 54, (2016), pp. 65-74.

[21] S. Zhou, L. Mi, H. Chen and Y. Geng, "Building detection in Digital surface model", 2013 IEEE International Conference on Imaging Systems and Techniques (IST), (2012). 
[22] J. He, Y. Geng and K. Pahlavan, "Toward Accurate Human Tracking: Modeling Time-of-Arrival for Wireless Wearable Sensors in Multipath Environment", IEEE Sensor Journal, vol. 14, no. 11, (2014), pp. 3996-4006.

[23] Z. Lv, A. Halawani and S. Fen, "Touch-less Interactive Augmented Reality Game on Vision Based Wearable Device", Personal and Ubiquitous Computing, vol. 19, no. 3, (2015), pp. 551-567.

[24] G. Bao, L. Mi, Y. Geng, M. Zhou and K. Pahlavan, "A video-based speed estimation technique for localizing the wireless capsule endoscope inside gastrointestinal tract”, 2014 36th Annual International Conference of the IEEE Engineering in Medicine and Biology Society (EMBC), (2014).

[25] D. Zeng and Y. Geng, "Content distribution mechanism in mobile P2P network", Journal of Networks, vol. 9, no. 5, (2014), pp. 1229-1236.

[26] W. Gu, Z. Lv and M. Hao, "Change detection method for remote sensing images based on an improved Markov random field", Multimedia Tools and Applications, (2015), pp. 1-16.

[27] Z. Chen, W. Huang and Z. Lv, "Towards a face recognition method based on uncorrelated discriminant sparse preserving projection", Multimedia Tools and Applications, (2015), pp. 1-15.

[28] M. Zhou, G. Bao, Y. Geng, B. Alkandari and X. Li, "Polyp detection and radius measurement in small intestine using video capsule endoscopy", 2014 7th International Conference on Biomedical Engineering and Informatics (BMEI), (2014).

\section{Authors}

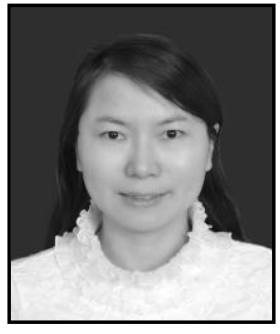

Wen Xiongjun, she received her M.S. degree in Intelligent system from Central South University in Hunan, China. Currently, she is a lecturer in the school of information science and engineering, Hunan International Economics University, Changsha, Hunan, China. Her research interest is mainly in the area of Software Technology, Network and Intelligent System, She has published several research papers in scholarly journals in the above research areas and has participated in several books. 